\title{
The absence of efficient dual pairs of spanning trees in planar graphs
}

\author{
T. R. Riley and W. P. Thurston * \\ Mathematics Department, 310 Malott Hall, Cornell University, Ithaca NY 14853-4201, USA \\ tim.riley@math.cornell.edu, wpt@math.cornell.edu
}

Submitted: Dec 7, 2005; Accepted: Aug 18, 2006; Published: Aug 25, 2006

2000 Mathematics Subject Classification: 05C10, 05C12, 20F06, 57M15

\begin{abstract}
A spanning tree $T$ in a finite planar connected graph $G$ determines a dual spanning tree $T^{*}$ in the dual graph $G^{*}$ such that $T$ and $T^{*}$ do not intersect. We show that it is not always possible to find $T$ in $G$ such that the diameters of $T$ and $T^{*}$ are both within a uniform multiplicative constant (independent of $G$ ) of the diameters of their ambient graphs.
\end{abstract}

\section{Introduction}

Suppose $G$ is a finite connected undirected graph (or multigraph) embedded in the plane. Given a spanning tree $T$ in $G$, define $T^{*}$ to be the spanning tree in the dual graph $G^{*}$ whose edges are those dual to edges in $G \backslash T$. Figure 1 gives an example.

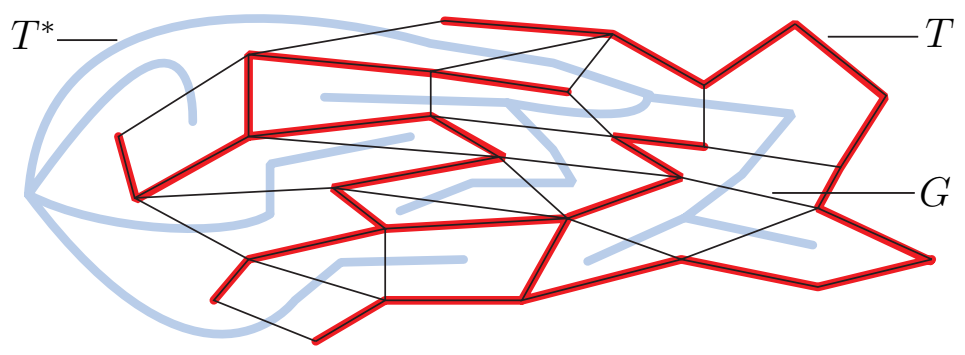

Figure 1: Dual spanning trees.

The length of a walk in a graph is the number of edges it contains and the distance between two vertices is the length of the shortest walk between them. The diameter

*The authors gratefully acknowledge support from NSF grants DMS-0540830 and DMS-0513436. 
Diam $G$ of a finite connected graph $G$ is the maximum distance between pairs of vertices of $G$.

Motivated by issues arising in Geometric Group Theory concerning the geometry of van Kampen diagrams, Gersten \& Riley asked [3]:

Question 1. Does there exists $C>0$ such that if $G$ is a finite connected planar (multi-) graph then there is a maximal tree $T$ in $G$ with

$$
\begin{aligned}
\operatorname{Diam} T & \leq C \operatorname{Diam} G, \text { and } \\
\operatorname{Diam} T^{*} & \leq C \operatorname{Diam} G^{*} ?
\end{aligned}
$$

They conjectured positive answers to a number of variants of this question with bounds imposed on the degrees of vertices in $G$ or $G^{*}$. We exhibit a family of graphs resolving these negatively.

Theorem 2. There are families $\left(G_{n}\right)_{n \in \mathbb{N}}$ of finite connected planar graphs such that all vertices in $G_{n}$ and $G_{n}^{*}$ have degree at most 6 , and there are constants $C_{1}, C_{2}>0$ such that for all $n \in \mathbb{N}$ and all spanning trees $T$ in $G_{n}$,

$$
\begin{aligned}
\operatorname{Diam} G_{n}+\operatorname{Diam} G_{n}^{*} & \leq C_{1} n, \text { and } \\
\operatorname{Diam} T+\operatorname{Diam} T^{*} & \geq C_{2} n^{2} .
\end{aligned}
$$

Establishing (2) involves two key ideas. The first is to regard $G_{n}$ as the 1-skeleton of a combinatorial 2-disc $\Delta_{n}$ and invoke a concept known as filling length. In the context of a simply connected metric space, Gromov [5] defined the filling length of a based loop $\gamma$ to be the infimal $L$ (assuming it exists) such that $\gamma$ can be contracted through a family of based loops each of length at most $L$ to the constant loop (i.e. to the basepoint). We will use a combinatorial analogue of filling length from [2] concerning shellings of diagrams.

A diagram $(\Delta, \star)$ is a finite planar contractible combinatorial 2-complex $\Delta$ equipped with a base vertex $\star$ on its boundary. One can regard $\Delta$ as a finite planar multigraph $G$, the 1-skeleton of $\Delta$, with a 2-cell filling each face other than the outer (i.e. unbounded) face. Define the boundary walk of $\Delta$ based at $\star$ to be the anti-clockwise closed walk around the boundary of $\Delta$ that has origin $\star$ and follows the attaching map of the outer face. The length of the boundary walk is the number of edges it contains (note that those in 1-dimensional portions of $\Delta$ are counted twice), or equivalently the degree of the vertex of $G^{*}$ dual to the outer face of $G$.

A shelling of a diagram $\Delta=\Delta^{0}$ down to a vertex $\star$ on its boundary is a sequence $\left(\Delta^{i}\right)_{i=0}^{m}$ of diagrams in which $\Delta^{m}$ is the single vertex $\star$ and, for all $i$, we obtain $\Delta^{i+1}$ from $\Delta^{i}$ by one of the following two moves.

- Remove a pendent edge and incident leaf $v \neq \star$.

- Remove an edge $e$ and the interior of a (closed) 2-cell $f$ where $e$ is in the boundaries of both $f$ and $\Delta^{i}$. 
Each such move results in an elementary homotopy of the boundary walk: in the first case a backtracking pair of edges is removed, and in the second $e$ is replaced by the complementary portion of the walk around the boundary of $f$. These moves ultimately achieve the contraction of the boundary walk of $\Delta$ down to the trivial walk at $\star$. So we define the filling length $\mathrm{FL}(\Delta, \star)$ of $(\Delta, \star)$ to be the minimal $L$ such that there is a shelling $\left(\Delta^{i}\right)_{i=0}^{m}$ of $\Delta$ in which for all $i$, the length of the boundary walk of $\Delta^{i}$ is at most $L$.

Filling length will be useful to us because, given a diagram $(\Delta, \star)$ with $G$ the 1 -skeleton of $\Delta$, the layout of a spanning tree $T$ in $G$ and the corresponding $T^{*}$ in $G^{*}$ can be made to dictate a shelling of $\Delta$ with filling length bounded above in terms of Diam $T+\operatorname{Diam} T^{*}$ (see Proposition 3). So a lower bound on the filling length of $(\Delta, \star)$ leads to a lower bound on Diam $T+\operatorname{Diam} T^{*}$.

This brings us to the second key idea, which is to construct diagrams $\left(\Delta_{n}, \star\right)$ so as to contain a fattened tree that forces the filling length of $\left(\Delta_{n}, \star\right)$ to be suitably large. In the context of Riemannian 2-discs this has been done by Frankel \& Katz in [1], answering a question of Gromov; our $\Delta_{n}$ will essentially be combinatorial analogues of their metric discs. To obtain $\Delta_{n}$ we first inductively define a family of trivalent trees $\mathcal{T}_{n}$ by taking $\mathcal{T}_{0}$ to be a lone edge, and $\mathcal{T}_{n}$ to be three copies of $\mathcal{T}_{n-1}$ with a leaf of each identified. (We note that this does not determine $\mathcal{T}_{n}$ uniquely.) We then fatten $\mathcal{T}_{n}$ to a complex $A_{n}$ (see Figure 2) in which each of its edges becomes an $n \times n$ grid. Finally, to obtain $\Delta_{n}$ we attach a combinatorial hyperbolic skirt (a planar 2-complex $B_{n}$ that is topologically an annulus - see Figure 3) around the boundary of $A_{n}$ to reduce the diameter of its 1-skeleton to $\sim n$.

Imagine inscribing $\mathcal{T}_{n}$ in the plane, circling it with a loop, and then contracting that loop down to a point. In the course of being contracted, the loop will intersect $\mathcal{T}_{n}$. In Lemma 4 we show that however the loop contracts it must, at some time, meet at least $n+1$ distinct edges of $\mathcal{T}_{n}$. Envisage $A_{n}$ to be inscribed with a copy of $\mathcal{T}_{n}$ as in Figure 2 . The lemma can be applied to the boundary walks of the diagrams $\Delta_{n}^{i}$ of any shelling of $\Delta_{n}$ to learn that for some $i$ at least $n+1$ distinct edges of $\mathcal{T}_{n}$ will be intersected; it then follows from the construction of $\Delta_{n}$ that at that time the length of the boundary walk is $\Omega\left(n^{2}\right)$.

Acknowledgement. Question 1 was a topic of class discussion in a course taught by the second author at Cornell University in the Fall, 2005. We are grateful to the members of the class, particularly John Hubbard and Greg Muller, for their contributions. Additionally, we thank Andrew Casson, Genevieve Walsh and two anonymous referees for their comments on earlier versions of this article.

\section{Constructing the graphs $G_{n}$}

Let $A_{n}$ be the family of diagrams (fattened trees) obtained from $\mathcal{T}_{n}$ (shown underlying) as illustrated in Figure 2 by replacing edges by $n \times n$ grids and non-leaf vertices by tessellated triangles.

For $k=2^{m}$ with $m \geq 3$ define $D_{k}$ to be the planar combinatorial 2-complex that is topologically an annulus and is built out of $m-2$ concentric rings of pentagons as shown 

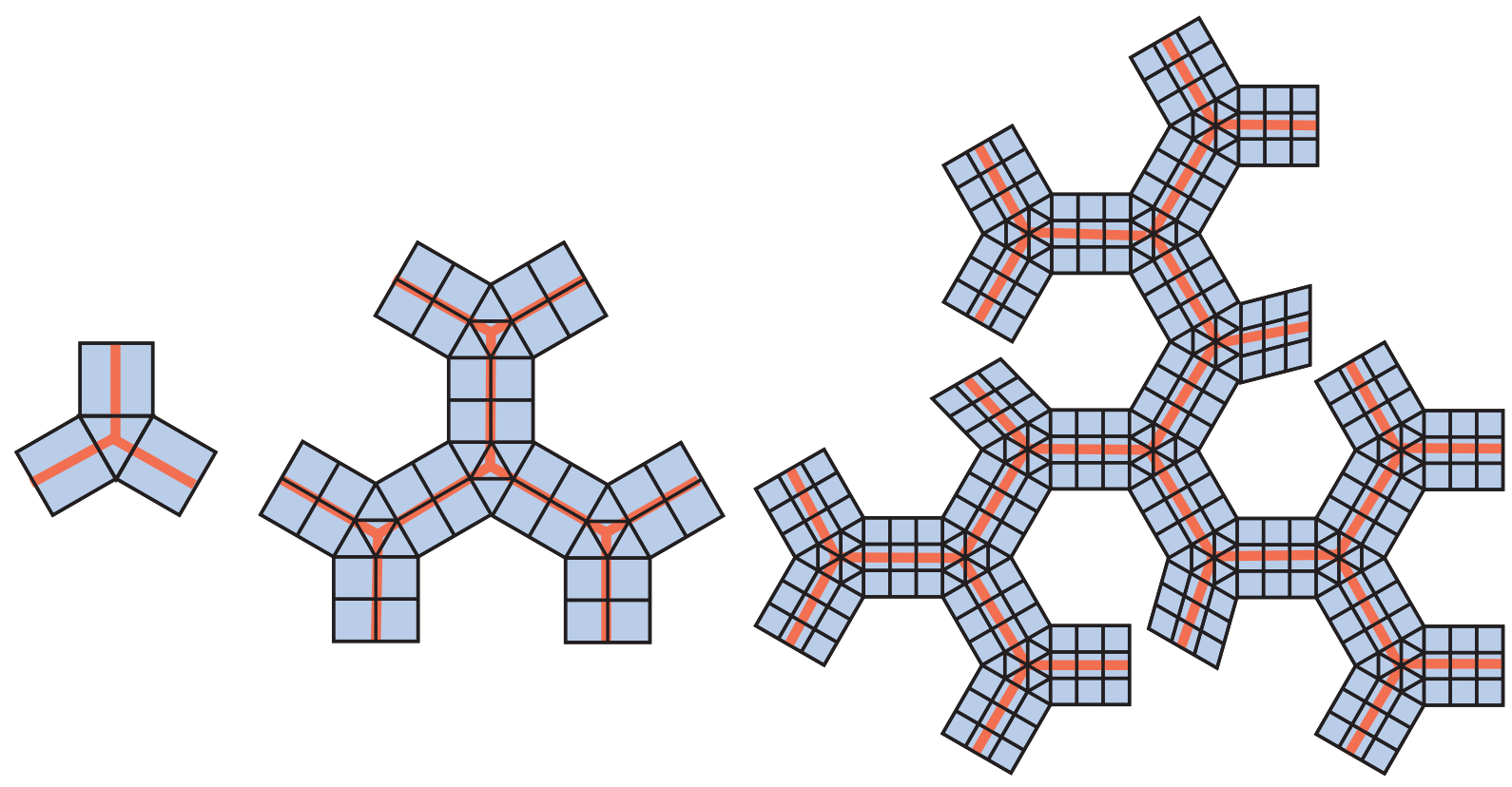

Figure 2: $A_{1}, A_{2}$ and $A_{3}$ inscribed with $\mathcal{T}_{1}, \mathcal{T}_{2}$ and $\mathcal{T}_{3}$.

in Figure 3 for $m=3,4,5$. For $m \geq 3$ and $2^{m-1}<k \leq 2^{m}$, obtain $D_{k}$ from $D_{2^{m}}$ by inserting single edges in place of pairs of adjacent edges sharing a degree-two vertex until the total number of edges in the outer boundary cycle is reduced to $k$. Figure 3 shows the example of $D_{44}$.
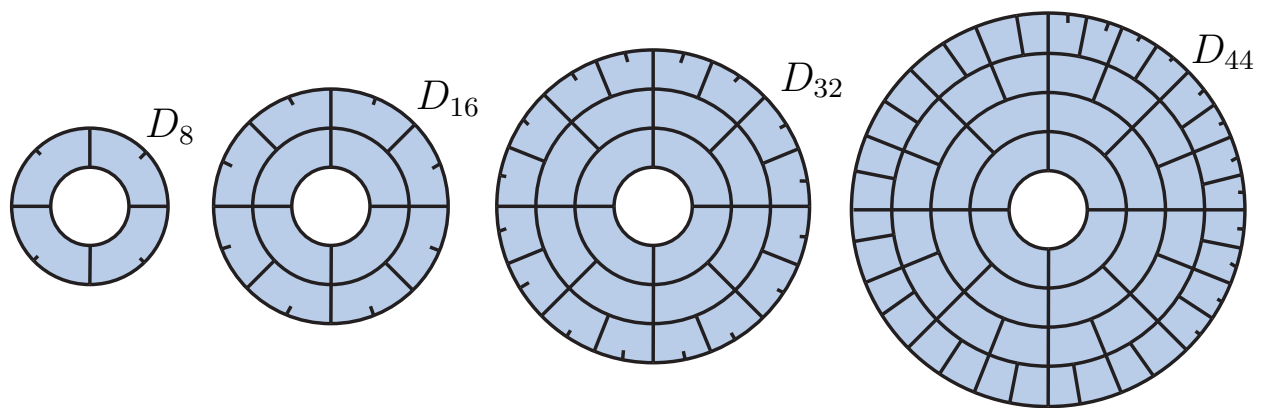

Figure 3: The annular 2-complexes $D_{k}$.

The combinatorial length of the boundary circuit of $A_{n}$ is $p_{n}:=\left(5.3^{n}+3\right) n / 2$. For $n \geq 1$, define $B_{n}:=D_{p_{n}}$, which plays the role of a hyperbolic skirt: attach $A_{n}$ to $B_{n}$ by identifying the boundary of $A_{n}$ with the outer boundary circuit of $B_{n}$ to give the planar combinatorial 2-disc $\Delta_{n}$. Let $G_{n}$ be the 1-skeleton of $\Delta_{n}$. 


\section{Diameter estimates}

We will now show that $\left(G_{n}\right)_{n \in \mathbb{N}}$ enjoys the properties listed in Theorem 2. By inspection, every vertex in $G_{n}$ and $G_{n}^{*}$ has degree at most 6 . Every vertex in $A_{n}$ is a distance at most $(n-1)$ from the boundary, and one checks that the diameter of $B_{n}$ is at most a constant times $n$ since the number of concentric rings is $O\left(\log p_{n}\right)$. Combined with similar considerations for the dual graphs this shows that there exists $C_{1}$ for which (1) holds.

For (2) we will use the following inequality from [4] on filling length. (In fact, the definition of a shelling used in $[2,4]$ allows a third move, omitted from our the definition in Section 1, but that move is not needed here and plays no role in the proofs of the results cited in this article, namely Propositions 3 and 5.)

Proposition 3 (Proposition 3.4, [4]). Suppose $(\Delta, \star)$ is a diagram in which the degree of each 2-cell is at most $\lambda$. If $T$ is a spanning tree in the 1-skeleton of $\Delta$ then

$$
\operatorname{FL}(\Delta, \star) \leq \operatorname{Diam} T+2 \lambda \operatorname{Diam} T^{*}+\ell(\partial \Delta)
$$

where $\ell(\partial \Delta)$ denotes the length of the boundary walk of $\Delta$.

We refer the reader to [4] for a detailed proof, but will sketch the idea here. Regard the vertex of $T^{*}$ outside $\Delta$ as the root $r$ of $T^{*}$. The embedding of $T^{*}$ in the plane defines a cyclic ordering on its leaves. Define a $T^{*}$-gallery of $\Delta$ to be a subcomplex that is the union of the closed 2-cells of $\Delta$ that are dual to the vertices lying on a path in $T^{*}$ from $r$ to a leaf. The idea is that tunnelling along paths of $T^{*}$ from $r$ to successive leaves, following their cyclic ordering, dictates a shelling $\left(\Delta^{i}\right)$ of $\Delta$ that establishes (3): when traversing an edge $e^{*}$ in such a path shell the edge $e$ dual to $e^{*}$ and the face dual to the terminal vertex of $e^{*}$; en route, remove all pendant edges (with leaf vertices $\neq \star$ ) immediately they become available. The boundary walks of the diagrams $\Delta^{i}$ are then each comprised of a path in $T$, trails in the 1-skeleta of two $T^{*}$-galleries of $\Delta^{i}$, and a portion of the boundary walk of $\Delta$. Thus we get (3).

For the following lemma and subsequent discussion it is convenient to regard $\mathcal{T}_{n}$ as a disjoint union of its edges; accordingly choose one edge in $\mathcal{T}_{n}$ to include both of its end-vertices and all others to include exactly one end-vertex.

Lemma 4. Suppose $\mathcal{T}_{n}$ is embedded in a disc, which for convenience we take to be the unit disc in the complex plane. Suppose $H:[0,1]^{2} \rightarrow \mathbb{D}^{2}$ is a continuous map (a homotopy) satisfying $H(0, t)=H(1, t)=1$ for all $t$, and $H_{0}(s)=e^{2 \pi i s}$ and $H_{1}(s)=1$ for all $s$, where $H_{t}$ denotes the restriction of $H$ to $[0,1] \times\{t\}$. Further, assume $H([0,1] \times[0, t]) \cap$ $H([0,1] \times[t, 1])=H([0,1] \times\{t\})$ for all $t$. Then $H_{t}$ meets at least $n+1$ edges in $\mathcal{T}_{n}$ for some $t \in[0,1]$.

Proof. The case $n=0$ is immediate. For the induction step, express $\mathcal{T}_{n}$ as the wedge

$\bigwedge_{i=1}^{3} \mathcal{T}_{n-1}^{i}$ of three copies of $\mathcal{T}_{n-1}$ at a vertex $v$. Obtain $\hat{\mathcal{T}}_{n-1}^{i}$ from $\mathcal{T}_{n}^{i}$ by removing a small open neighbourhood of $v$. Let $t_{i}$ be such that $H_{t_{i}}$ meets at least $n$ edges of $\hat{\mathcal{T}}_{n-1}^{i}$. Renumbering if necessary, we may assume $t_{1} \leq t_{2} \leq t_{3}$. The condition that $H([0,1] \times$ 
$[0, t]) \cap H([0,1] \times[t, 1])=H([0,1] \times\{t\})$ for all $t$, ensures that if $t_{1} \leq t \leq t_{3}$ and $H_{t}([0,1]) \cap\left(\mathcal{T}_{n-1}^{1} \cup \mathcal{T}_{n-1}^{3}\right)=\emptyset$ then points of $H_{t_{1}}([0,1]) \cap\left(\mathcal{T}_{n-1}^{1} \cup \mathcal{T}_{n-1}^{3}\right)$ are in different path components than points $H_{t_{3}}([0,1]) \cap\left(\mathcal{T}_{n-1}^{1} \cup \mathcal{T}_{n-1}^{3}\right)$ in $\mathbb{D}^{2} \backslash H_{t_{3}}([0,1])$, but that is impossible as $\mathcal{T}_{n-1}^{1} \cup \mathcal{T}_{n-1}^{3}$ is path connected. We deduce, in particular, that $H_{t_{2}}$ intersects $\mathcal{T}_{n-1}^{1} \cup \mathcal{T}_{n-1}^{3}$, and so meets at least $n+1$ edges of $\mathcal{T}_{n}$.

We can now establish (2). Choose any vertex on the boundary of $\Delta_{n}$ to serve as the base vertex $\star$. Envision the subdiagram $A_{n}$ of $\Delta_{n}$ to be inscribed with $\mathcal{T}_{n}$ as in Figure 2. The diagrams $\Delta_{n}^{i}$ of a shelling of $\left(\Delta_{n}, \star\right)$ are subcomplexes whose boundary walks define concentric loops ultimately contracting to $\star$. Interpolating suitably between these loops produces a homotopy in which the boundary walk of $\Delta_{n}$ is contracted to the constant loop at $\star$ through a family of loops $H_{t}$. So by Lemma 4 there exists $t$ such that $H_{t}$ meets $n+1$ edges of $\mathcal{T}_{n}$ and it follows that there exists $i$ such that the boundary walk of $\Delta_{n}^{i}$ meets $n+1$ edges of $\mathcal{T}_{n}$. But any path in the 1-skeleton of $\Delta_{n}$ meeting four distinct edges of $\mathcal{T}_{n}$ has combinatorial length at least $n$. So the length of the boundary walk of $\Delta_{n}^{i}$ is at least $n\lfloor n / 3\rfloor$. Deduce that $\operatorname{FL}\left(\Delta_{n}, \star\right) \geq n\lfloor n / 3\rfloor$ and therefore, by Proposition 3, there exists $C_{2}>0$ such that (2) holds.

\section{Two concluding remarks}

We note that Proposition 3.3 in [4] exhibits another family of diagrams in which filling length outgrows 1-skeleton diameter. However, filling length does not outgrow the diameter of the dual in these examples.

Finally, we mention that our family of diagrams $\Delta_{n}$ exhibits the most radical divergence possible between filling length, diameter and dual diameter in the sense of the following result.

Proposition 5. Given $\lambda>0$, there exists $C=C(\lambda)$ such that if $(\Delta, \star)$ is a diagram in which the degree of each 2-cell is at most $\lambda$ then

$$
\operatorname{FL}(\Delta, \star), \leq C(\operatorname{Diam} G)\left(\operatorname{Diam} G^{*}\right),
$$

where $G$ is the 1-skeleton of $\Delta$.

This follows from an argument of [2] which we will only briefly outline here. Take a geodesic spanning tree $T$ in $G$ based at * - that is, a spanning tree such that for all vertices $v$ in $G$, the distance from $v$ to $\star$ in $T$ is the same as in $G$. Note that $\operatorname{Diam} T \leq 2 \operatorname{Diam} G$. Let the vertex $r$ of $G^{*}$ that is outside $\Delta$ be the root of $T^{*}$. By subtrees suspended from a vertex $v$ in $T^{*}$ we mean the closures of the connected components of $T^{*} \backslash\{v\}$ that do not contain $r$. Describe a vertex as branching when there is more than one subtree suspended from it. A vertex below $v$ is any vertex of any subtree suspended from $v$. Define the weight of a tree to be the number of vertices it contains that have degree at least three.

Consider tunnelling through $\Delta$ along the walk in $T^{*}$ that starts at $r$, first proceeds to the nearest leaf or branching vertex (possibly $r$ itself) and then continues according to the following rules from its current vertex $v$. 
- If $v$ is a branching vertex then of the as-yet-unentered subtrees suspended from $v$, choose one of least weight and proceed to the nearest leaf or branching vertex $(\neq v)$ therein.

- If $v$ is a leaf return to the most recently visited branching vertex attached to which there remain as-yet-unentered suspended subtrees of $T^{*}$.

The walk is complete when every edge in $T^{*}$ has been traversed. This walk dictates the following shelling of $\Delta$ (termed logarithmic shelling in [2]): when traversing an edge $e^{*}$ for the first time, remove the dual edge $e$ and the face dual to the terminal vertex of $e^{*}$, and immediately any pendant edge (with leaf vertex not $\star$ ) appears, remove it.

The lengths of the boundary walks of the diagrams encountered in this shelling are at most a constant (depending on $\lambda$ ) times $(\operatorname{Diam} T) \log (1+$ Area $\Delta)$, where Area $\Delta$ denotes the number of 2-cells in $\Delta$. As Area $\Delta \leq \lambda^{\operatorname{Diam} G^{*}}$ and $\operatorname{Diam} T \leq 2 \operatorname{Diam} G$, the result follows.

\section{References}

[1] S. Frankel and M. Katz. The Morse landscape of a Riemannian disc. Ann. Inst. Fourier, Grenoble, 43(2):503-507, 1993.

[2] S. M. Gersten and T. R. Riley. Filling length in finitely presentable groups. Geom. Dedicata, 92:41-58, 2002.

[3] S. M. Gersten and T. R. Riley. Some duality conjectures for finite graphs and their group theoretic consequences. Proc. Edin. Math. Soc., 48(2):389-421, 2005.

[4] S. M. Gersten and T. R. Riley. The gallery length filling function and a geometric inequality for filling length. Proc. London Math. Soc., 92(3):601-623, 2006.

[5] M. Gromov. Asymptotic invariants of infinite groups. In G. Niblo and M. Roller, editors, Geometric group theory II, number 182 in LMS lecture notes. Camb. Univ. Press, 1993. 